\title{
SEP 91963
}

Design Studies of Proton Linear Accelerators ${ }^{*}$

D. E. Young, R, S. Christian, C. D. Curtis, T. W. Edwards, F. J. Kriegler, F. E. Mills, P. L. Morton, D. A. Swenson, J. Van Bladel

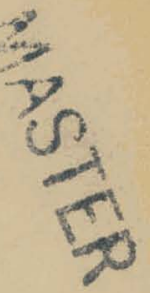

Midwestern Universities Research Association, Stoughton, Wisconsin

\section{INTRODUCTION}

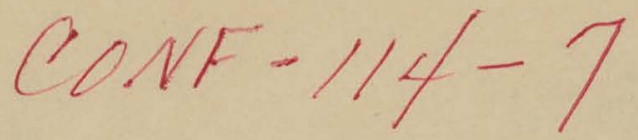

Design studies of a $200 \mathrm{Mev}$ conventional standing wave type of proton

linear accelerator to be used as an injector for a 12.5 Gev FFAG accelerator have been carried out at MURA. The standing wave linear accelerator is limited in energy to about the value mentioned because of the decreasing efficiency of this structure at higher energies. However, because of the high efficiency at energies less than this, the ease of extraction, and the favorable external beam quality, the standing wave linac is receiving wide attention not only from synchrotron injector designers, but also from groups interested in continuing the linear acceleration with a change of cavity structure into the meson energy region. Groups known to be active in the design of standing wave proton linear accelerators of about this energy include groups at Brookhaven, Yale University, Los Alamos, Berkeley, Saclay, CERN, and Harwell.

In the last couple of years the art of design has been advanced by extensive use of digital computation techniques. At least two major problems are amenable to the computer approach. The first is an investigation of the radio-frequency structure. At MURA a program has been written which calculates the frequency and fields, and any other related quantity, from Facsimile Price \$ Microfilm Price $\$$

*Supported by the U. S. Atomic Energy Commission. 


\section{DISCLAIMER}

This report was prepared as an account of work sponsored by an agency of the United States Government. Neither the United States Government nor any agency Thereof, nor any of their employees, makes any warranty, express or implied, or assumes any legal liability or responsibility for the accuracy, completeness, or usefulness of any information, apparatus, product, or process disclosed, or represents that its use would not infringe privately owned rights. Reference herein to any specific commercial product, process, or service by trade name, trademark, manufacturer, or otherwise does not necessarily constitute or imply its endorsement, recommendation, or favoring by the United States Government or any agency thereof. The views and opinions of authors expressed herein do not necessarily state or reflect those of the United States Government or any agency thereof. 


\section{DISCLAIMER}

Portions of this document may be illegible in electronic image products. Images are produced from the best available original document. 
the geometry ppecified--arbitrary shaped drift tubes with holes are possible, but azimuthel symmetry is required. A similar program exists at Harwell ${ }^{1}$ and other laboratories are writing or using such programs. The Yale group calculates resonant geometry, but in this case the fields are specified from charge distributions and the geometry is generated from the fields. ${ }^{2}$ The second use of digital techniques is to study the particle dynamies in the linear accelerator. At MURA a program is in use which allows a large number of particles (500) to be simultaneously "flushed" through a linac which has previously been described to the computer in terms of accelerating fields, geometry, drift spaces, and quadrupole focusing fields. Output can be obtained at any place along the linac so that it is possible to study the linac performance in terme of beam quality as the linac design is varied. This represents a powerful technique for studying the linac particle dynamics in the presence of the forces which couple the phase motion to the tranaverse motion. It provides a means for evaluating the fabrication tolerances, the effect of drift spaces between cavities, the problem of matching the linac injector into the linac, the optimum quadrupole focusing field strength, and many other aspects of linac design.

It appears that it is possible to design an adequate standing wave siructure to accelerate particles to $200 \mathrm{Mev}$ and to optimize the structure from the standpoint of cost. This optimization is based on the fact that. excluding fixed costs, the linac cost (including operating costb) can be broken down into costs per unit peak ri power and coste per unit length of accelerator. The cost of $r$ power for a given acceleration can be reduced 
by achleving a large value of $2 T^{2}$ of the cavity structure, where $Z$ is the shunt irrpedance and $T$ is the transit time factor. However, if $Z T^{2}$ is made large at the expense of the length of the cavity, that is to say, if a drift tube shape is chosen whtch is more likely to spark so that the acceleration rate riust be decreased. the saving in power cost will be offset by the increased length cost. The sparking limit imposes a boundary condition on the maximum electric field in the cavity.

A few other design boundary conditions might be mentioned. The tank lengths are dictated by the power requirements which can be met by a single power amplifier tube. Sufficient reserve power must be avallable to allow compensation for beam loading and for practical cavity losses. At the same time the tank lengths cannot be so large that tank flattening is difficult, a restriction that prevents lengths of greater than 20 wavelengths. The drift tubes must not only be shaped to give a maximum $\mathrm{ZT}^{2}$ and a maximurn safe electric field close to 15 million volts per meter, but must allow radial focusing devices to be incorporated. The drift-tube holes must allow for the beam radial acceptance and for misalignments. A frequency of 200 megacycles seems a good choice although this does impose difficult restrictions onthe early oirift tuines.

There is an obvious need for more experimental informaton to allow a practical design to be chosen, eapecially in the higher energy region. Cost savings should be possible by investigating new fabrication techniques in the cevities and drift tubes. The problems associated with the higher energy in the inac mut be studied, especially with regard to exciting, tuning, and 
phase controlling the ffeld, dissipating the increased power, and establishIng better limits for the sparking levels. At the present time drift-tube stems and tuners which do not have azimuthal symmetry cannot be handled in the computing programs so that such frequency perturbations must be measured. There is a striking need for more and better instrumentation to allow a more informed tune up and operation to be achieved. With a many tank system it is no longer realigtic to depend on simply knob furning to maximize the beam.

\section{A 200 MEV LINAC DESIGN}

Figures 1 and 2 illustrate a typical design of a $200 \mathrm{Mev}$ linac broken down Into tank lengths which can be powered by a 5 megaiwatt power stage and which are less than 20 wavelongths long. The geometry has been chosen using cylindrical shaped drift tube although some advantage may be realized by using some other drift-tube shaps. The average axial accelerating fleld has been chosen so that the peak electric field in any tank is conservatively below the limit Imposed by sparking considerations.

In this design special effort has been made to achleve a geometry (using cylindrically shaped drift tubes) which results in a cost minimum. By asseasing both capital and operating power costs and capital and operating length costs, it is posstble to evaluate the cost of the structure per Mev which stays within the limit imposed by sparking. In order to evaluate the structure in this way, it is necessary to have information for a broad range of the geometrical parameters, 1, e., tank diameters, drift-tube shapes, gap lengths. Ueing the MURA field computational program, to be described 
in the next section, a large number of computer rung heve been made for resonant geometry close to 200 megacycles and at energies of $50,100,150$, and $200 \mathrm{Mev}$, and with a smaller number of runs at $10 \mathrm{Mev}$ intervals from 10 to 200 Mev. These runs give values for the transit time factor, the power efficiency measured in terms of the product of the shunt impedance and the square of the transit time factor, and the peak field normalized to unit average axial accelerating field. From this data, cost curves can be developed for any specified geometry. A representative set of curves is shown in FIgs. 3, 4, and 5. These figures show the cost of the structure as a function of the drift-tube diameter for varlous radii of curvature on the corner of a cylindrical drift tube and at a constant tank diameter. For cylindirlcel drift tubes of small diancter, the values of both $2 \mathrm{~T}^{2}$ and the peak electric field are large. In order to offset the larger peak fields, the cavity excitation must be reluced, thus lowering the average accelerator rate and increasing the length of the linac, and the costs associated with length. When the hole is included in the drift tube, an obvious lower limit is imposed on the drift tube diameter. For the cost curves shown, the cost minimum at 200 Mev occurs for a cavity diameter of 84 centimeters and a drift-tube corner radius of 6 centimeters (for \& 4.5 centineter hole diameter vith some curvature on the hole corner).

The cost minimum is quite broad allowing some flexibility in the delft-tube deglgn. Since our extensive study has includsd only the four energies mentioned, the design shown in Figs. 1 and 2 may not be considered optimum throughout even fo: a cylinarical drift-tube shape. Also the 
requirement of using a constant tank diameter may result in some departure from the optimum over the energy range covered by the tank.

\section{FIELD COMPUTATIONS}

The mathematical detalls of the MURA field computational program are given in the Appendix. The geometry of the unit cell is specifled in terms of a tank diameter (D), unit cell length (L), gap length (g), and drift-tube contour. Cylindrical shaped drift tubes specified by a diameter (d), a corner radius $\left(R_{c}\right)$, a hole diameter $(a)$, and a hole corner radius $\left(R_{\mathrm{HC}}\right)$, have received the greatest attention, but other shapes are also possible. In particular. ellipsoidal drift tubes, specified by a major and minor axis and a hole diameter with a hole corner radius, have been selected to achieve higher pover efficiencles than exhibited by the cylindrical shapes. A few more arbitrarily shaped drift tubes have been considered, but only a few computer runs have been made of these shapes.

Having specified the geometry of the unit cell, the computer program calculates a resonant frequency and the flelds at every point. From the fields it is not difficult to calculate the quantities of interest in luac design, namely, the power dissipated to the outer walls, the end plates, and the dxift tube, the Q, the shunt impedance, the transit time factor, or any other quantity based on these azimuthally symmetric fields. In particular, it is posstble to search the metallic aurfacen for the maximum electric fleld to see where sparking may occur and at what level. By renormalizing the fields to the maximum. peak eiectric field considered "safe" from sparking considerations, the average axial accelerating field is found so that particle energy gain and the 
corresponding power dissipated can be calculated. This allows the geometry to be evaluated on a practical basis.

This program has been used to investigate the geometry at energies of $50,100,150$, and $200 \mathrm{Mev}$ for cylindrical drift tubes. Resonance curves have been developed which allow the tank diameter, drift-tube diameter, gap length, and drift-tube corner radius to be chosen for resonance at 200 megacycles at these energles. Further, an examination of these runs allows one to make a selection of the geometry which will result in the largest power efficiency. Figure 7 shows the maximum shunt impedance (including the transit sime factor' plotted as a function of energy. It may not be possible to choose values this large in practice, however, Eince the hole size and the necessity to stay within the sparlsing limit without unduly increasing the linac length imposes other restrictions. Shown in the same figure is a curve showing the possible gain in $\mathrm{ZT}^{2}$ in going to an ellipsoidal shape for the drift tube. Again it is not claimed that the additional gain in $\mathrm{ZT}^{2}$ for the ellipsoldal shape can be reallzed in practice. We have also considered drift tubes of other shapes such as that seen in Fig. 12, but no extensive study has been carried out for these cases and in fact appear to be undesirable from peak accelerating voltage considerations.

Figures 8 through 13 show field plots for various drift-tube shapes which are resonant at 200 megacycles. The $F$ field $\left(F=r H_{\phi}\right.$, where $r$, $\Sigma$ and $\phi$ are the usual cylindrical coordinates so that $\mathrm{H}_{\phi}$ is into the plane of the paper) indicates the direction of the electric field which has been divided into 15 increments, the raximum being along the axis. By dividing 
the $F$ field by $r$, the iso-H lines are obtained and may help the reader to ascertain where the large currents are flowing in the cell. The following values apply:

Cylindrical Ellipsoldal

\begin{tabular}{lcc}
$\mathrm{Z}$ (shunt impedance) & 51.2 & 52.2 \\
$\mathrm{~T}$ (transit time factor) & 0.59 & 0.67 \\
$\mathrm{ZT}^{2}$ & 17.6 & 23.4 \\
\hline
\end{tabular}

The gain in power efficiency for the ellipsoidal drift tube is attributed to the increased transit time factor. This applies as well to the odd shaped drift tube shown.

At the present time nearly 1000 computer runs have been made at MURA for the type of geometry shown. These rung are now being sumriarized and published as an aid in the design of standing wave linear accelerators.

\section{PHASE AND RADIAL MOTION IN LINACS}

We have prepared a computer progranj to trace the phase motions and the transverse motion of particles through a proton linear accelerator. The program is prepared to simulate a linac of standard design, namely a linac composed of a set of resonant linac cavities (tanks), each containing a series of drift tubes, each of which contains a quadrupole focusing magnet.

The program proceeds by transforming the coordinates of a collection of particles through a set of transformations representing the linac. 
The transformations are chosen to represent the important effects of the linac accelerating and focusing structure on the particles.

Each particle has gix coordinates, namely $x, x^{\prime}, y, y^{\prime}, E$ and $\phi$. where $x, x^{\prime}, y$, and $y^{\prime}$ are the transverse displecements and angles of the particle trajectories in two transverse directions, $E$ is the energy and $\phi$ is the phase of the particle with respect to the $r f$ accelerating voltage. If one ignores the coupling between the $x, x^{\prime}$ motion, the $y, y^{\prime}$ motion and the E, $\phi$ motion, one can study each of the three motions Independently. This has bees done on many occasions, both analytically and digitally and is well descrtbed in the Lterature. $3,4,5,6$

It is extremely difficult to include the coupling between the transverse motion and the phase motion in the analytic approach. The present work is an attempt to study the effect of these coupling terms with the aid of digital computation.

In order to describe the nature of the coupling terms included in the program, we must outline the sequences of transformation performed by the program, and describe the functional dependence of each transformation.

The linac is treated as a series of cells, each cell beginning at the center of one drift tube and ending at the center of the next. The main loop of the program transforms the ccordinates of the particles through one cell:

The first transformation is denoted on the slide as $\mathrm{T} 1$ and simulates the action of the quadrupole magnet on the transverse coordinates $x, x^{\prime}, y$ 
and $y^{\prime}$. The $x, z^{\prime}$ motion is independent of the $y, y^{\prime}$ and $\phi$ coordinates. but is a function of the particle energy $E$. This is one of the terms which couple the transverse motions to the phace motion.

The second transformation is denoted on the alide as $\mathrm{T} 2$ and simulates a free-space dxift to the electricel center of the accelerating gap. There are no coupling terms present here.

Before proceeding to the tranglormation T3 at the center of the gap, Wre shall dispense with transformation $\mathrm{T} 4$ and $\mathrm{T} S \mathrm{by}$ noting that they are Aimilar, except in magnitude, to transformations $\mathrm{T} 2$ and $\mathrm{T} 1$ respectively.

The transformation $\mathrm{T} 3$ at the center of the gap simulates the change in energy of the particle, and the net defocusing experienced by the particle on crossing the gap.

The change in $\phi$ from the previous gap is a simple function of the length of the cell and the longitudinal component of the particle velocity. The difference between the actual velocity, and the fongitudinal component is a possibie coupling botween the phase motion and the transverse motion. This effect is very small and is ignored.

The change in energy on crossing the gap is a function of $\phi$ and a longitudins transit time function. The longitudinal trans it time is a function of the particle energy $\mathbb{E}$, and the effective length of the gap, which, in turn, is a function of the transverse coordinates $y$ and $y$. Fence the change in energy 19 a function of $\phi, E, x$ and $y$. This represents a strong coupling . . between the phase motion and the transverse motion. 
The defocusing action on the $\mathrm{z}^{\prime}$ coordinate of a particle on crossing the gap is a function of $\phi$ and $x$ and a transverse transit time function. which is a function of $E, x$, and $y$.

We are in the process of studying the admittance and emittance of spectif linac structures with specific values for the quadrupole field strengthe. From the same study will come information on the nonadiabaticity of the phase motion in the low energy end of the linac. Many other aspects of linac degign will be studied with this program.

\section{CONCLUSION}

The design of gtanding wave proton linear accelerators has been aided greatly in the last fow years with the extensive use of digital computational techniques. Field computational programs allow a detailed investigation of the $r$ structure even to the extent of a cost optimization. Particle dynamics programs will yleld better information and insight into particle behavior in linacs whose parameters can be readily adjusted. There is a need however for better experimental information, especially in the energy range above $50 \mathrm{Mev}$. 


\section{APPENDIX}

Mathematical Procedures of the Field Computational Program

The calculation of electromagnetic elgenvectorg and eigenvalues in linear accelerator-type cavitles containing stemless drift.tubes whose meridian plane cross sections are plecewise simply describable, can be accomplished by solving finite difference approximations to the wave equation. ${ }^{8}$ The resulting elgenvectors and eigenvalues allow quantitative determination of relevant auxillary electromagnetic quantities and of particle dynamic phenomena.

Maxwell's equations, when expressed in cylindrical coordinates, admit two linearly and azimuthally independent sets of solutions; one with a vanishing $E_{4}$, the other with a vanishing $\mathrm{H}_{\phi}$ (TM or TE modes, respectively). The former type of solution or mode has a nonvanishing $E_{z}$ and hence permits an axial acceleration of particles, whereas the latter type does not.

Thus the modes of interest are the TM modes, for which:

$$
E_{z}=\frac{i}{u \cdot E} \frac{1}{r} \frac{\partial\left(r \bar{H}_{\phi}\right)}{\partial r}, \quad E_{r}=\frac{-1}{w E} \frac{\partial\left(H_{\phi}\right)}{\partial z} \text {. }
$$

and

$$
\frac{1}{\mathbf{r}} \frac{\partial}{\partial \mathbf{r}}\left(\mathbf{r} \frac{\partial \mathrm{H}}{\partial \mathbf{r}}\right)+\frac{\partial^{2} \mathrm{H}_{\phi}}{\partial z^{2}}+\left(\mathrm{k}^{2}-\frac{1}{\mathbf{r}^{2}}\right) \mathrm{H}_{\phi}=0 .
$$

such that

$$
\mathrm{k}^{2}=\omega^{2}, i \epsilon=\frac{\mu^{2}}{\mathrm{c}^{2}}=\left(\frac{2 \pi}{\lambda}\right)^{2} .
$$

The boundary condition applicable to the solution of Eq. (2) must be such that the tangential component of $E$ vanishes on all metallic surfaces and 
is expressable as:

$$
\epsilon \frac{\partial}{\partial t}(\hat{n} \times \bar{E})=\hat{n} \times(\bar{V} \times \bar{H})=0 .
$$

where upor:

$$
\hat{\mathbf{n}} \cdot \bar{\nabla} \cdot\left(\mathbf{r} \mathrm{H}_{\phi}\right)=\frac{i}{\partial \mathrm{n}}\left(\bar{r} \cdot \mathrm{H}_{\phi}\right)=0 .
$$

Because of the comparative simplicity of the boundary condition,

Eq. (5), it has been found advantageous to formulate the problem in terms of the dependent variable $F=r{ }^{H} \phi$, whereupon the field components, Eq. (1), and the scalar wave equation, Eq. (2), become:

$$
E_{z}=\frac{i}{\omega \epsilon} \frac{1}{r} \frac{\partial F}{\partial r}, \quad \quad i_{r}=\frac{-i}{i i \epsilon} \frac{1}{r} \frac{\partial F}{\partial z} .
$$

and

$$
\frac{\partial^{2} r}{\partial r^{2}}-\frac{1}{r} \frac{\partial^{2}}{\partial r}+\frac{\partial^{2} F}{\partial z^{2}}+k^{2} F=0 .
$$

At the onset of numerical solution of Eq. (7), subject to the boundary condtion, Eq. (5), neither the eigenvector, $F$, nor the eigenvalue, $k$, are known. However, any "trial eigenvector", $\phi$, can always be expanded in terms of the true eigenvectors, $\phi_{n^{\prime}}$ in the form:

$$
\phi=\sum_{n} A_{n} \phi_{n} \quad \text { where } \mathcal{K}_{n}+k^{2} \phi_{n}=0 \text {. }
$$

and where, in the present inetance $f$ is the operator indicated in Eq.. (2).? Upon manipulating the scalar product of $₫$ with $f$; the lowest eigenvalue $k_{1}$ can be expressed as:

$k_{1}^{2} \leqslant-\frac{\langle\phi, \phi \phi\rangle}{\langle\phi, \phi\rangle}=\frac{-\iint_{Q} \frac{F}{\bar{r}}\left[\frac{\partial^{2} F}{\partial x^{2}}-\frac{1}{r} \frac{\partial F}{\partial r}+\frac{\partial^{2} F}{\partial z^{2}}\right] d r d z}{\iint_{s} \frac{F^{2}}{r} d r d z}$. 
It is furthermore demonstrable that this leads to a variational principle, such that if $\phi$ differs from the lowest eigenvector $\phi_{1}$ by a small amount proportional to a parameter $\lambda$, then the inequality of Eq. (8) 18 proportional to $\lambda^{2}$. This may be done by expanding the ratio of scalar products in a Taylor serles and noting that the term proportional to $\lambda$ vanishes, if the operator is self-adjoint, such that:

$$
\frac{\langle\phi, \rho \phi\rangle}{\langle\phi, \phi\rangle}=\frac{\left\langle\left(\phi_{1}+\lambda \psi\right), \rho\left(\phi_{1}+\lambda \psi\right)\right\rangle}{\left\langle\left(\phi_{1}+\lambda \psi\right),\left(\phi_{1}+\lambda \psi\right)\right\rangle}=-\mathrm{k}_{1}^{2}+0\left(\lambda^{2}\right) .
$$

Thus a convergent iterative process is realized if, alternatively, better "trial eigenvectors" $\phi$ or $F$ are chosen, and successively better eigenvalue approximations are calculated.

The calculation of improved trial elgenvectors may conveniently be accomplished by the technique of (over)relaxation. Upon constructing a cartesian two-dimensional array of discrete values of $F$ at vartous $r$ and $z$, the problem can be formulated as a "matrix" problem rather than the continuous problem expressed by Eq. (7). The fundamental equations, Eq. (7) and Eq. (9), may thus be written in finite difference form by expanding the various neighboring matrix elements about a central element $F_{i j}$ via Taylor series. If only the four nearest neighbors of the central element $F_{i j}$ are employed, the resulting approximations to Eq. (7) and Eq. (9), become, respectively:

$$
\begin{aligned}
F_{i, j+1}\left(1-\frac{h}{2 r_{j}}\right) & +F_{i, j-1}\left(1+\frac{h}{2 r_{j}}\right)+F_{1+1, j}+F_{1-1, j}+ \\
& +\left(k^{2} h^{2}-4\right) F_{i j}+O\left(h^{4}\right)=0,
\end{aligned}
$$




$$
k_{1}^{2} h^{2}=\frac{\sum_{1} \sum_{j}\left\{\frac{F_{j j}^{n}}{r_{j}}\left[F_{i, j+1}^{n}\left(1-\frac{h}{2 r_{j}}\right)+F_{i, j-1}^{n}\left(1+\frac{h}{2 r_{j}}\right)+F_{i+1, j}^{n}+F_{i-1, j}^{n}-4 F_{i j}^{n}\right] s_{i j}\right.}{\sum_{i} \sum_{j} \frac{\left(F_{i j}^{n}\right)^{2}}{r_{j}} \Delta s_{i j}},
$$

where $i$ and $j$ are matrix indices referring to the $z$ and $r$ coordinates, reapectively, and where $h$ is the common interelement coordinate difference. The finite difference equation, Eq. (11), may be written as a Liebmann four-point algorism:

$$
F_{i j}^{n+1}-F_{i j}^{n}=\alpha\left\{F_{i j}^{n}\left(\frac{k^{2} h^{2}}{4}-1\right)+\frac{1}{d}\left[F_{i, j+1}^{n}\left(1-\frac{h}{2 r_{j}}\right)+F_{i, j-1}^{n+1}\left(1+\frac{h}{2 r_{j}}\right)+F_{1+1, j}^{n}+F_{i-1, j}^{n+1}\right]\right\}
$$

where it is assumed that the matrlx is repetitively traversed in a regular manner, such that, upon improving $F_{i j}^{n}$ into $F_{i j}^{n+1}$, the matrix elements $F_{1-1, j}^{n+1}$ and $F_{i, j-1}^{n+1}$ will have already been processed on the current $(n+1)^{\text {th }}$ traversal, whereas the matrix elements $F_{i, j+1}^{n}$ and $F_{i+1, j}^{n}$ are those remaining from the previous $n^{\text {th }}$ traversal.

Note that the boundary condition given by Eq. (5) corresponds to a reflection effect which is easily applicable to all boundarles which coincide with a row or column of matrix elements. For example. $F_{t, j+1}^{n}$ can be replaced by $F_{i, j-1}^{n+1}$ in Eq. (13). However, for boundartes that are not parallel with a row or column of matrix elements, suoh a procedure is not easily accomplished in applying the requisite boundary condition. For this latter case, an aurillary "curvilinear matrix" can be constructed whtch follows the contour of the boundary and sufficiently overlaps the principal "rectangular 
matrix." The application of the boundary condition to this auxiliary matrix is then again easily accomplished by a reflection of the form indicated above.

The expressfons analogous to Eqs. (13) and (12), for a curvilinear matrix element, are:

$$
\begin{aligned}
& F_{i m}^{n+1}-F_{i m}^{n}=\alpha\left\{F_{i m}^{n}\left[\frac{k^{2}}{\left(\frac{2}{h_{p}^{2}}+\frac{2}{h_{\theta}^{2}}\right)}-1\right]+\frac{1}{\left(\frac{2}{h_{p}^{2}}+\frac{2}{h_{\theta}^{2}}\right)}\left[F_{l+1, m}^{n}\left\{\frac{1}{h_{\rho}^{2}}+\frac{1}{2 h_{\rho}}\left[\frac{r_{c}}{\rho\left(r_{c}+\rho \sin \theta\right)}\right]\right\}\right.\right. \\
& +F_{l-1, m}^{n+1}\left\{\frac{1}{h_{r}^{2}}-\frac{1}{2 h_{\rho}}\left[\frac{r_{c}}{\left(r_{c}+\rho \sin \theta\right)}\right]\right\}+F_{\ell, m+1}^{n^{\prime}}\left\{\frac{1}{h_{\theta}^{2}}-\frac{1}{2 h_{\theta}}\left[\frac{\cos \theta}{\left(r_{c}+\rho \sin \theta\right.}\right]\right\} \\
& \left.+F_{l, m-1}^{n+1}\left\{\frac{1}{h_{\theta}^{2}}+\frac{1}{2 h_{\theta}}\left[\frac{\cos \theta}{\left(r_{c}+p \sin \theta\right)}\right]\right\}\right] \text {, }
\end{aligned}
$$

and

$$
\begin{aligned}
& \sum_{l} \sum_{m} \int \frac{F_{m}^{n}}{\left(r_{c}+\rho \sin \theta\right)}\left[\frac{h^{2}}{h_{p}^{2}}\left(F_{l+1, m}^{n}+F_{l-1, m}^{n}\right)+\frac{h^{2}}{h_{\theta}^{2}}\left(F_{l, m+1}^{n}+F_{l, m-1}^{n}\right)+\right.
\end{aligned}
$$

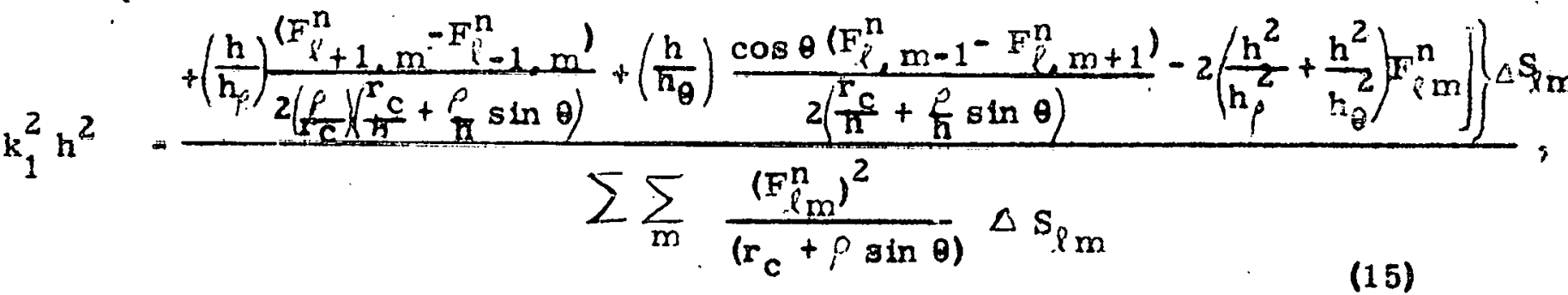

where $\rho$ is the (local) radius vector to the matrix element $F_{l_{m}}$ as defined in the curvilinear coordinate system, $\theta$ is the angle made by this radius vector with the axis of the cavity, $r_{c}$ is the radial coordinate (In the cartesian system) of the (ocal) center of curvature associated with the matrix element $F_{l m}$. and $h_{p}$ and $h_{\theta}$ are the radial and azimuthal interelement coordinate differences assoctated with the matrix element $F_{l m}$. 
The factor a appearing in Eq. (13) and Eq. (14) is the overrelaxation factor. If $i=1$, these algorisms are rigorous. By judtclous choice of $x \geqslant 1$, however, accelerated convergence may be obtained. Although some attempts have been made to calculate the optimum value of $\alpha$ for algorisms derivable from Laplace's equation, little information has been found in connection with wave equations such as Eq. (7). Empirically, a value between approximately 1.4 and 1.9 , increasing as the number of rectangular matrix elements increases from about 1000 to 8000 , has been found best.

Further, the factors $\Delta S_{i j}$ and $\Delta S_{P_{m}}$ appearing in Eq. (12) and Eq. (15) are meridian plane subareas required for the finite-difference Integrations ard associated with the various masrix elements $F_{i j}$ and $F_{l m}$; whereas the fact that all of the various matrix elements in Eq. (12) and in Eq. (15) are labeled with a superscript $n$ signifles that each eigenvalue improvement should be deduced from those matrix elements which exist at the end of a traversal of all elements (done for the purpose of matrix element improvement).

In order to make full use of both the cartesian matrix and the curvtLinear matrix, it is necessary to interpolate some matrix elements from one form of matrix to the other. Such interpolation formulae as are required may be deduced, iur_exarriple, by expanding five matrix elements $F_{i j}$ in two-dimensional Taylor sories about the matrix element $F_{\ell m}$, and requiring that a weighted sum of the flve Taylor series be consistent with the fundarrental identity, Eq. (7). This process leads, in this case, to: 
13

$$
F_{m}=\sum_{u=1}^{5} \lambda_{u}\left(F_{i j}\right)_{u} /\left(\sum_{u=1}^{5} \lambda_{u}-k^{2}\right)
$$

where the $\lambda$ 's are the solutions of the indicated $5 \times 5$ matrix problem.

In practice, it is advantageous to use the best Initial "trial eigenvector" available. Initially this may be done by using an analytic approximation, whereas, afte: a repertoire of solutions for geometries of interest has been developed, a reasonably good initial trial elgenvector may be obtained from a previously calculated solution.

The eigenvector 3 and eigenvalues so calculated rnay be used to compute several auxiliary electromagnetic quantitles, including:

Energy Stored per Untt Volume: $\quad \frac{W}{V}=\frac{\mu}{2} \frac{\int \frac{F^{2}}{r} d r d z}{\iint r \text { ir dz }}$ Power Lost to Cavity Walls:

$$
P_{w}=\pi R_{g} \int \frac{F^{2}}{r} d \dot{\varphi}
$$

Quality Factor:

$$
Q=\frac{i^{\prime} d}{R_{5}} \frac{\iint \frac{F^{2}}{r} d r d z}{\oint \frac{F^{2}}{r} d e}
$$

Average Axtal Accelerating Field: $\quad \xi_{0}=\frac{1}{\omega \epsilon I} \int\left(\frac{1}{r} \frac{\partial F}{\partial r}\right)_{0, z} d z$.

Shuni Impedance:

$$
z_{S}=\varepsilon_{0}^{2} /\left(P_{w} / L\right) \text {. }
$$

Transit Time Factor:

$$
T=\frac{1}{w \in \tau \varepsilon_{0}} \int\left(\frac{1}{r} \frac{\partial F}{\partial r}\right)_{0, z} \cos \frac{2 \pi z}{l} d z
$$

Coupling Coeffictent:

$$
S=\frac{1}{w \in L^{2} \varepsilon} \int_{0}\left(\frac{1}{r} \frac{\partial F}{\partial r}\right)_{0, z} z \sin \frac{2 \pi z}{L} d z
$$


It is convenient to normalize the eigenvector thus calculated, 80 as to permit eagy compartaon of results from similar geometry. This normalization may be carried out, for exariple, by requiring that the stored energy per unit volume or the average axial acceleraing fleld remain coustant. In thle way relative figures of merit may be obtained frotn which the best gcometry for a particular cavity energy, etc. , may be deterninid. 


\section{REFERENCES}

1. R. Taylor, J. Nuc. Energy, Part C. Vol. 3, p. 123 (1961).

2. R. L. Gluckstern, Proceediugs of the 1961 International Conference on High Energy Accelerators (Brookhaven National Laboratory, 1961), p. 129.

3. J. C. Slater, Rev. Miod. Phys. 20, 473 (1948).

4. J. P. Blewett, Internal BNL Report JPB-18 (March 27, 1963).

5. L. Smith and R. L. Gluckstern, Rev. Sc1. Ingtr. 26, 220 (1955).

6. D. Cohen, Internal ANL Report, ANLAD-57 (July 16, 1959).

7. J. Van Bladel, MURA Report No. 481 (1859, unpublished).

8. T. W. Edwards, MURA Report No. 622 (1961, unpublished). 


\section{FIGURE CAPTIONS}

Fig. 1. A 200 Mev Linear Accelerator Design.

Fig. 2. 200 Mev Linear Accelerator Parameters.

Fig. 3. A Cost Curve at $200 \mathrm{Mev}$ for a Tank Diameter of 64 Centimeters.

Fig. 4. A Cost Curve at $200 \mathrm{Mev}$ for a Tank Diameter of 76 Centimeters.

Fig. 5. A Cost Curve at $200 \mathrm{Mev}$ for a Tank Diameter of 84 Centimeters.

Fig. .6. Unit Cell Geometry (Cylindrical Drift Tube).

Fig. 7. 2T $\mathrm{T}^{2}$ as a Function of Energy for Cylindrical and Ellipsoidal Drift Tubes.

Fig. 8. Cylindrical F-Field (200 Mev).

Fig. 9. Cylindrical Iso-H Field (200 Mev).

Fig. 10. Ellpsoidal F-Field (200 Mev).

Fig. 11. Ellipsoldal Iso-H Fleld (200 Mev).

Fig. 12, Odd-Shaped Drift Tube F-Field (200 Niev).

Fig. 13. Odd-Shaped Drift Tuhe Iso-H Plot (200 Mev).

Fig. 14. Functional Dependence of Transformation. 


\begin{tabular}{|c|c|c|c|c|c|c|}
\hline & $\begin{array}{l}\text { Tank } \\
\text { No. } 1\end{array}$ & $\begin{array}{l}\text { Tank } \\
\text { No. } 2 \\
\end{array}$ & $\begin{array}{l}\text { Tank } \\
\text { No. } 3 \\
\end{array}$ & $\begin{array}{l}\text { Tank } \\
\text { No. } 4 \\
\end{array}$ & $\begin{array}{l}\text { Tank } \\
\text { No. } 5 \\
\end{array}$ & $\begin{array}{l}\text { Tank } \\
\text { No. } 6 \\
\end{array}$ \\
\hline Energy Range (Mev) & $0.75-10$ & $10-53$ & $53-95$ & $95-132$ & $132-167$ & $167-200$ \\
\hline Acceleration Rate ( $\mathrm{Mv} / \mathrm{m})$ & 1.7 & 1.7 & 1.7 & 1.6 & 1.4 & 1.3 \\
\hline Tank Length $(\mathrm{m})$ & 5.5 & 25 & 24.5 & 23.5 & 24.5 & 25.5 \\
\hline Tank Diameter $(\mathrm{m})$ & 0.94 & 0.94 & 0.92 & 0.88 & 0.86 & 0.84 \\
\hline $\begin{array}{l}\text { Approximate Drift } \\
\text { Tube Diameter }(\mathrm{cm})\end{array}$ & 18 & $19-13$ & $15-14$ & 18 & 19 & 19 \\
\hline $\begin{array}{l}\text { nmift Tube Radius of } \\
\text { Zurvature }(\mathrm{cm})\end{array}$ & - & 1 & 4 & 6 & 6 & 61 \\
\hline $\begin{array}{l}\text { Drift Tube Aperture } \\
\text { Diameter }(\mathrm{cm})\end{array}$ & 2.5 & 3.0 & 3.5 & 4.0 & 4.5 & 4.5 \\
\hline $\begin{array}{l}\text { Average Shunt Impedance } \\
\text { (including transit time) } \\
\text { (megohms/meter) }\end{array}$ & 65 & 50 & 38 & 25 . & 20 & 17 \\
\hline $\begin{array}{l}\text { Cavity RF Losses (mega- } \\
\text { watts) }\end{array}$ & 0.3 & 1.8 & 2.3 & 3.0 & 3.0 & 3.1 \\
\hline
\end{tabular}

Figure 1. A $200 \mathrm{Mev}$ Linear Accelerator Design. 
Tank Resonant Frequency:

Stable Phase Angle:

Total Accelerating Length:

Total RF Peak Power Required (with beam loading):

Average RF Power at $1.2 \%$ Duty Factor:

Number of Drift Tubes:

Maximum Fleld Strength at any Point less than $14 \mathrm{Mv} / \mathrm{m}$.
200 megacycles per second $64^{\circ}$

$128.5 \mathrm{~m}$

24 megawatts

290 Kilowatts

245

Figure 2. $200 \mathrm{Mev}$ Linear Accelerator Parameters. 


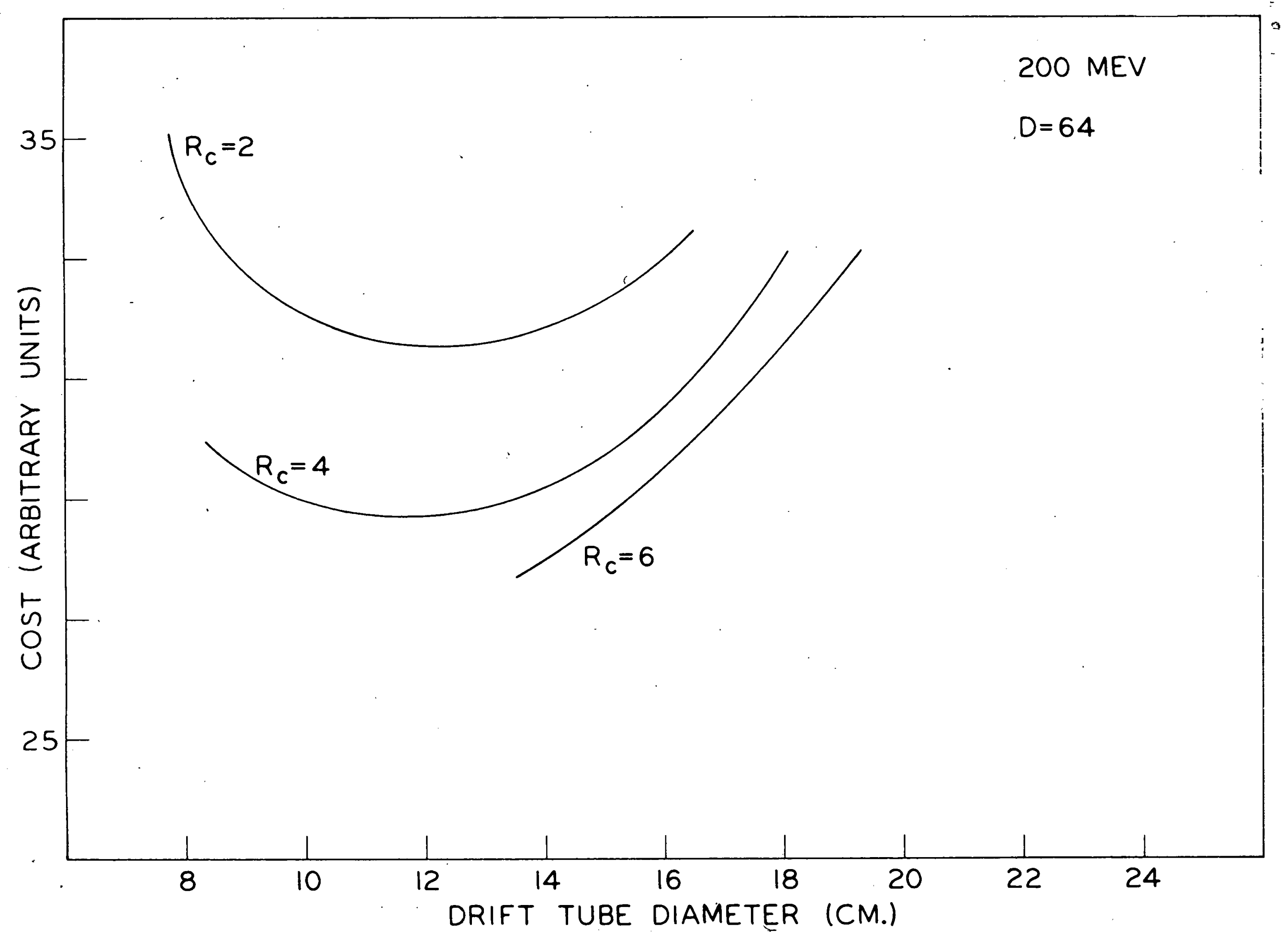




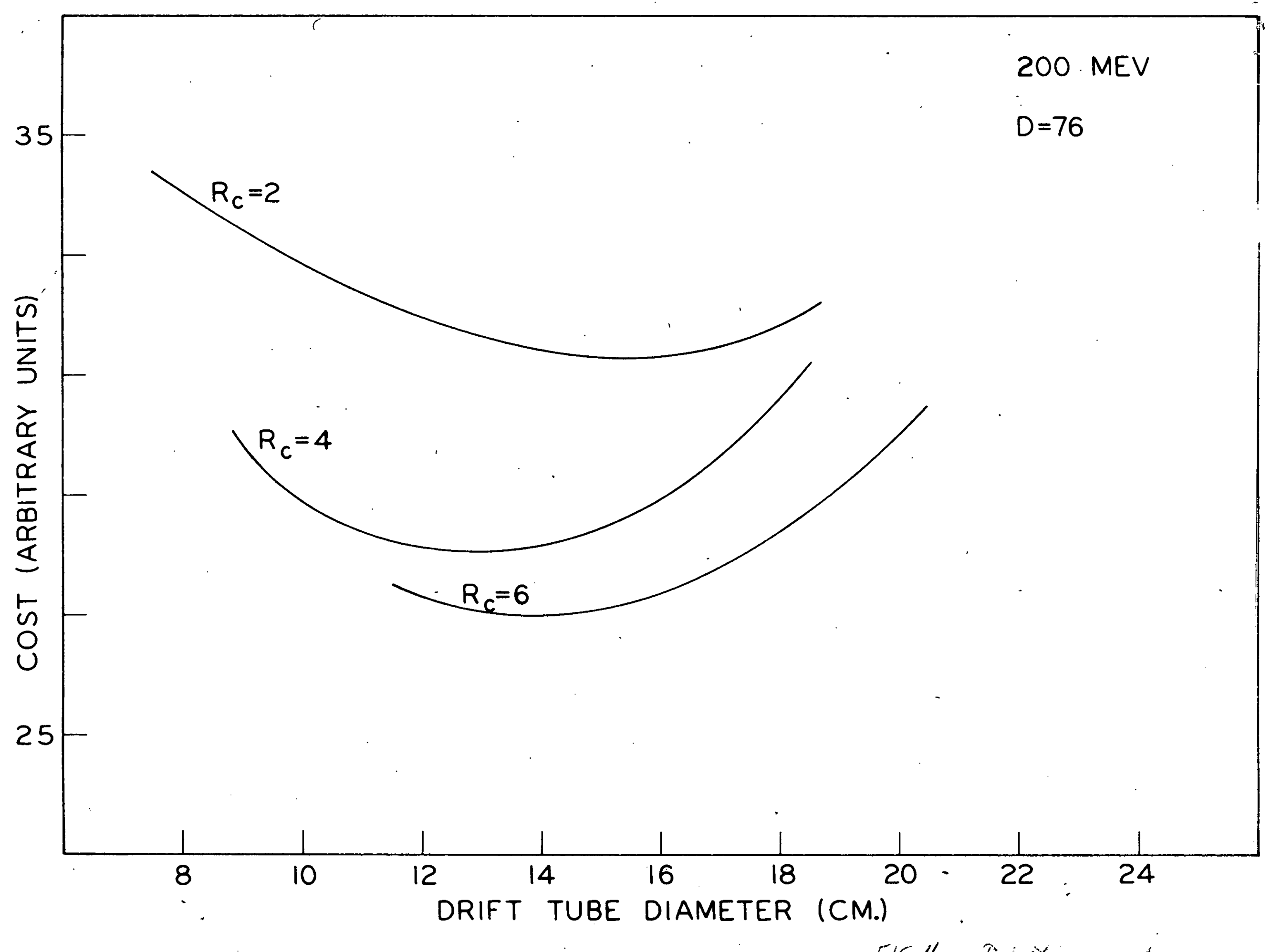






FIG 5 Oa 


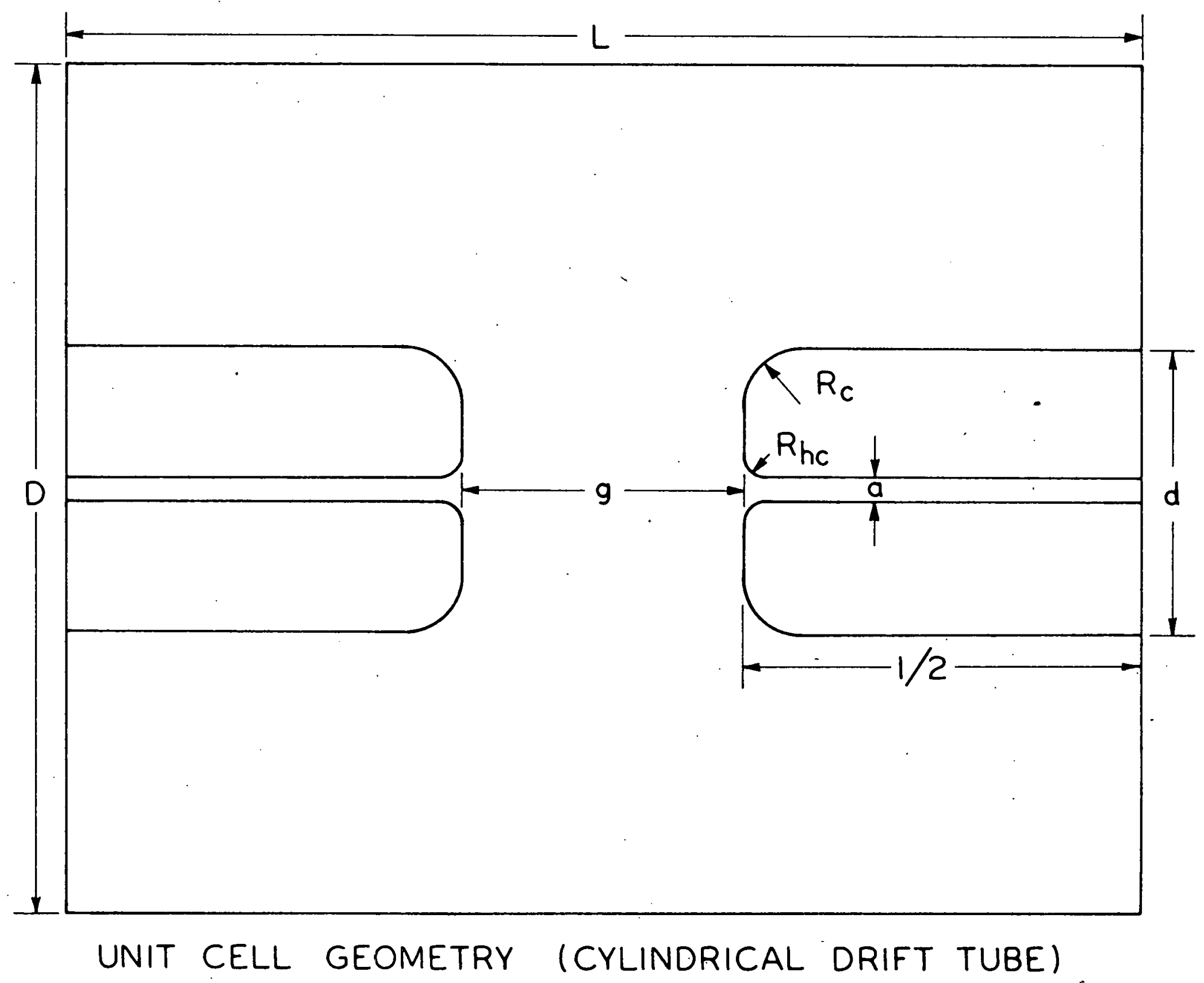

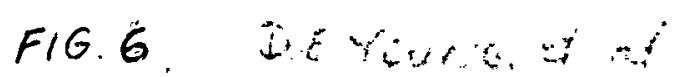




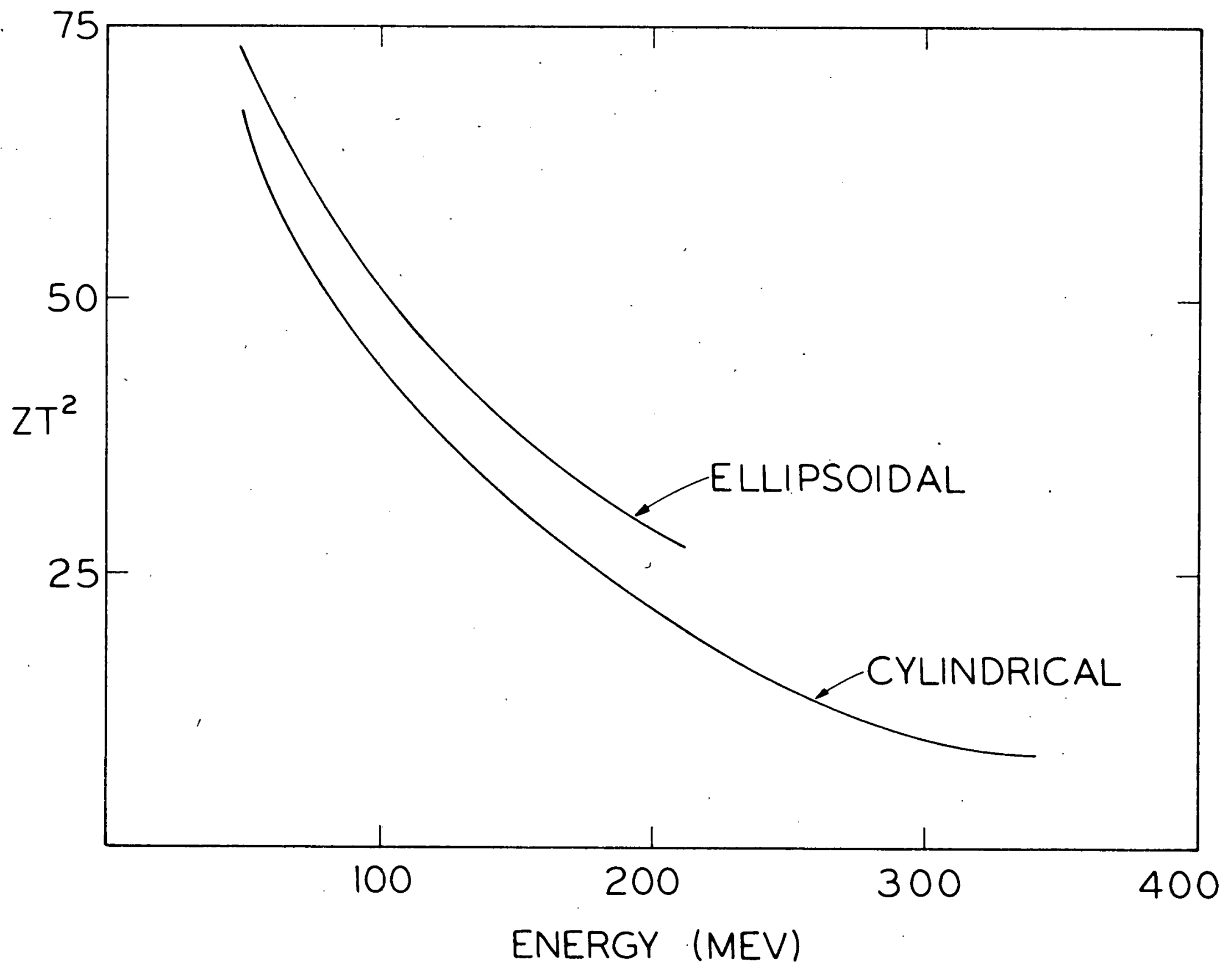

FIG $7 \quad 3: \because:$, 6 : 
CYLINDRICAL $\Gamma$ FIELD (200 MEV)

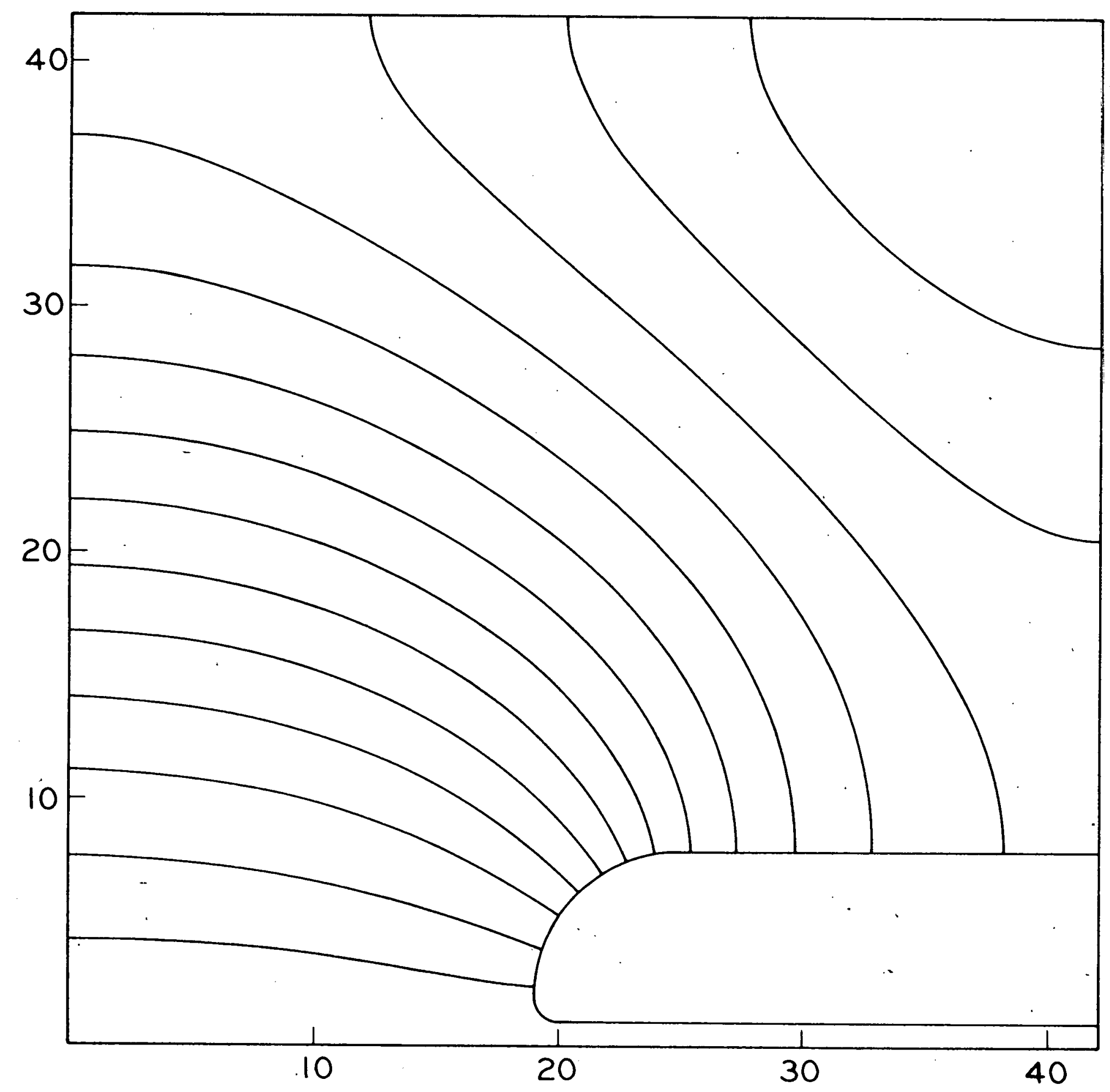


CYLINDRICAL - $)-H$ FIELD (200 MEV)

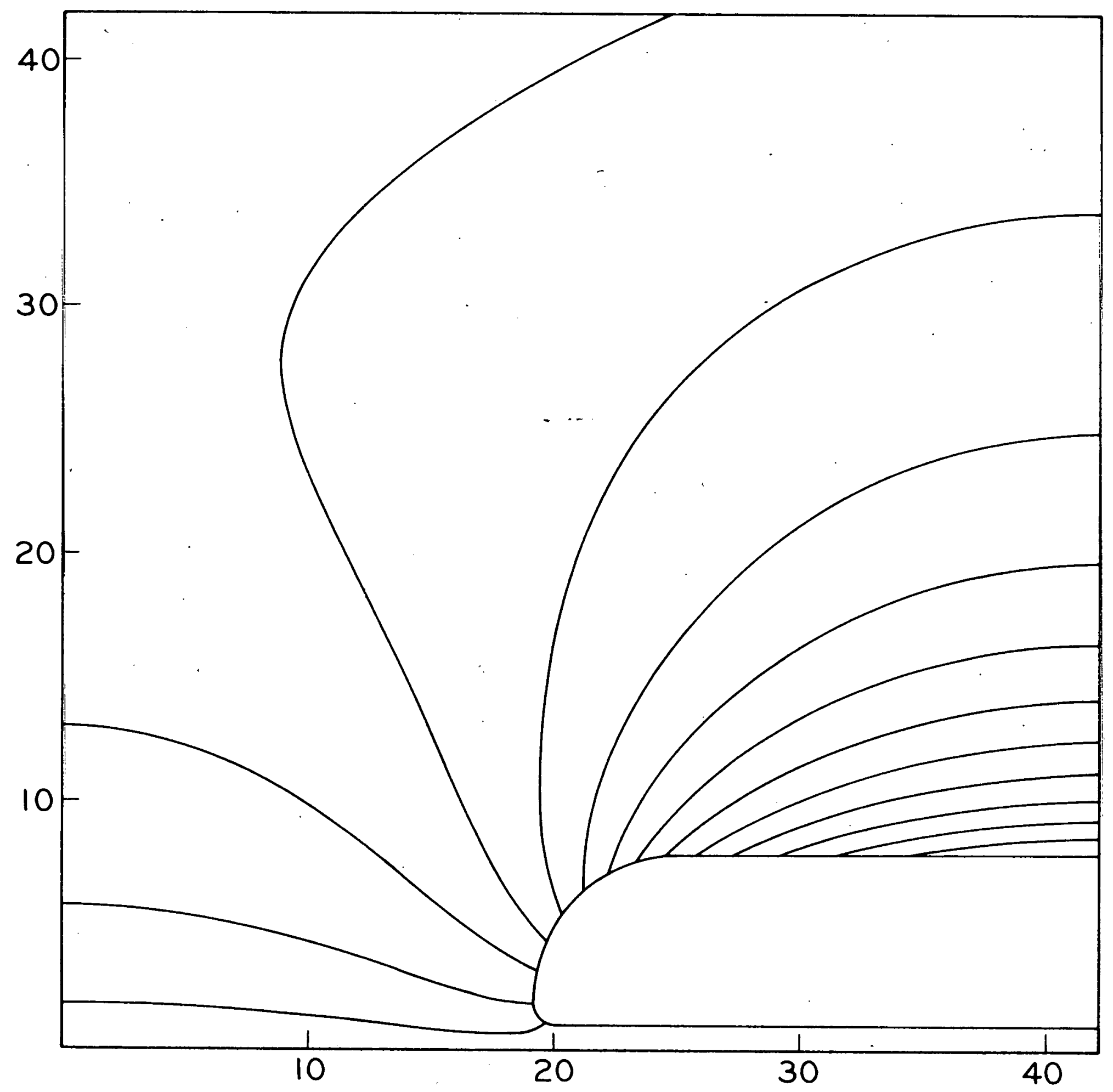


ELLIPSOID $\cdots$ F-FIELD (200 MEV)




ELLIPSOIDAL ISC ' + FIELD (200 MEV)






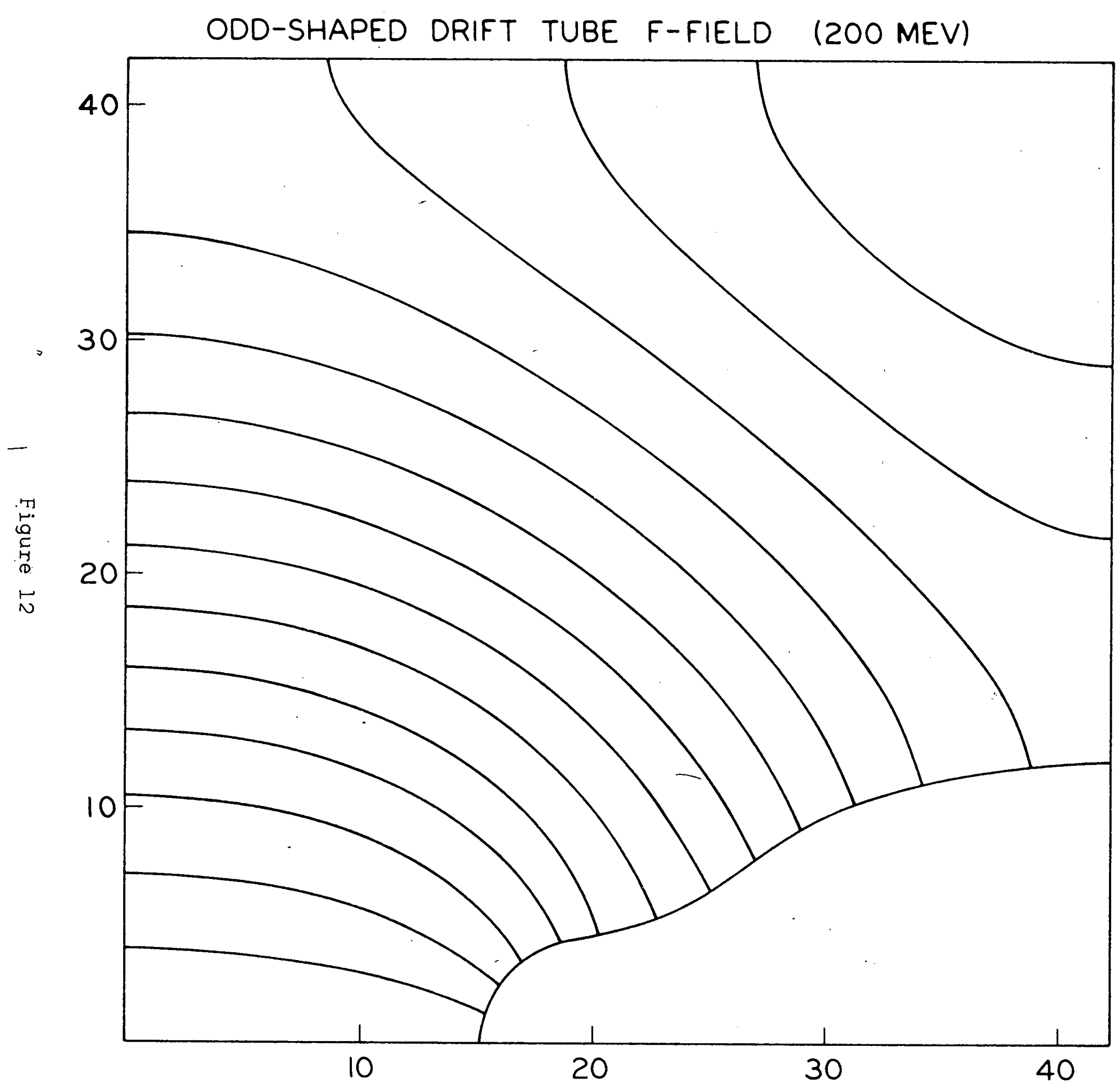


ODD-SHAPED :DRIFT TUBE ISO-H PLOT (200 MEV)

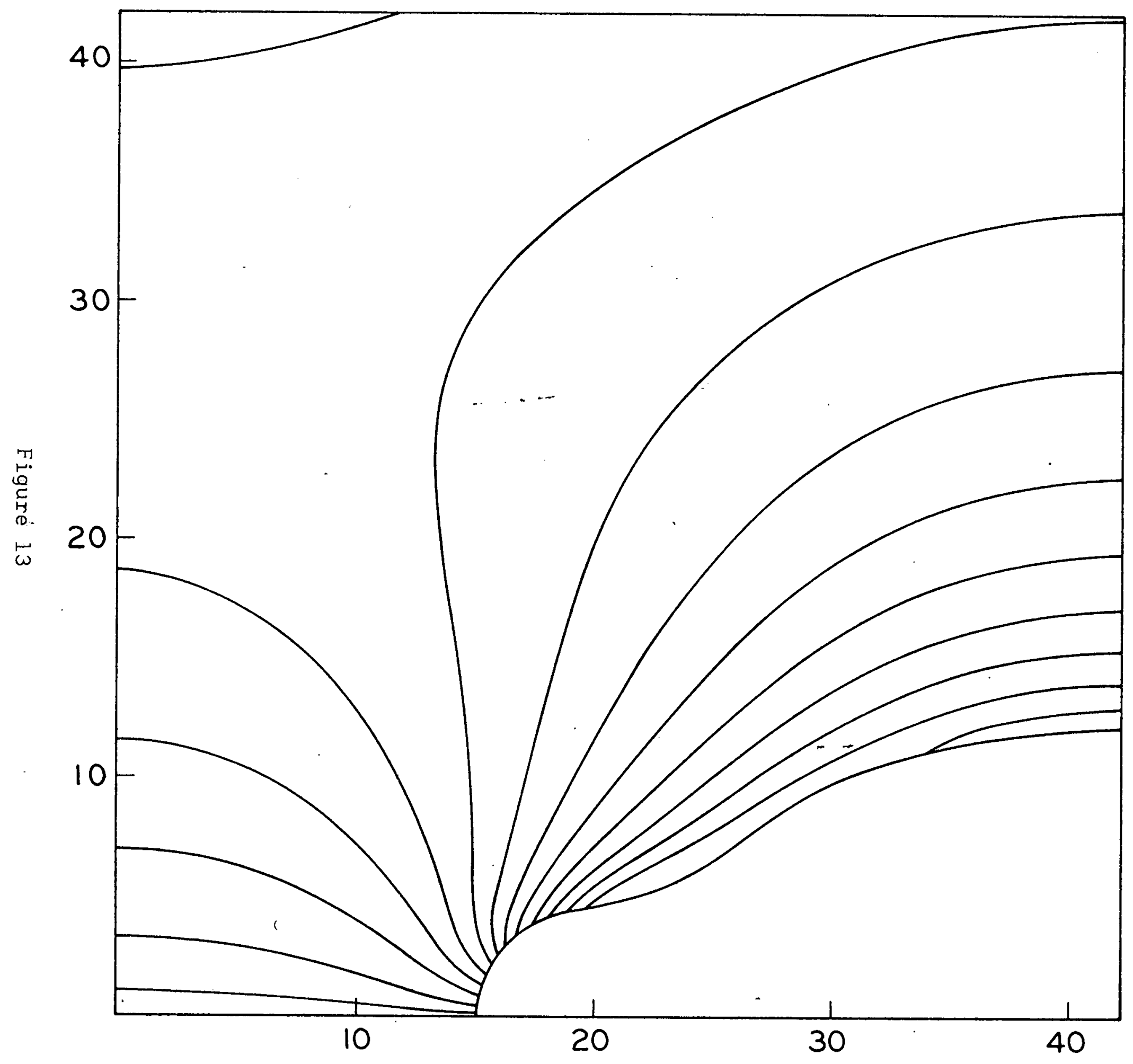




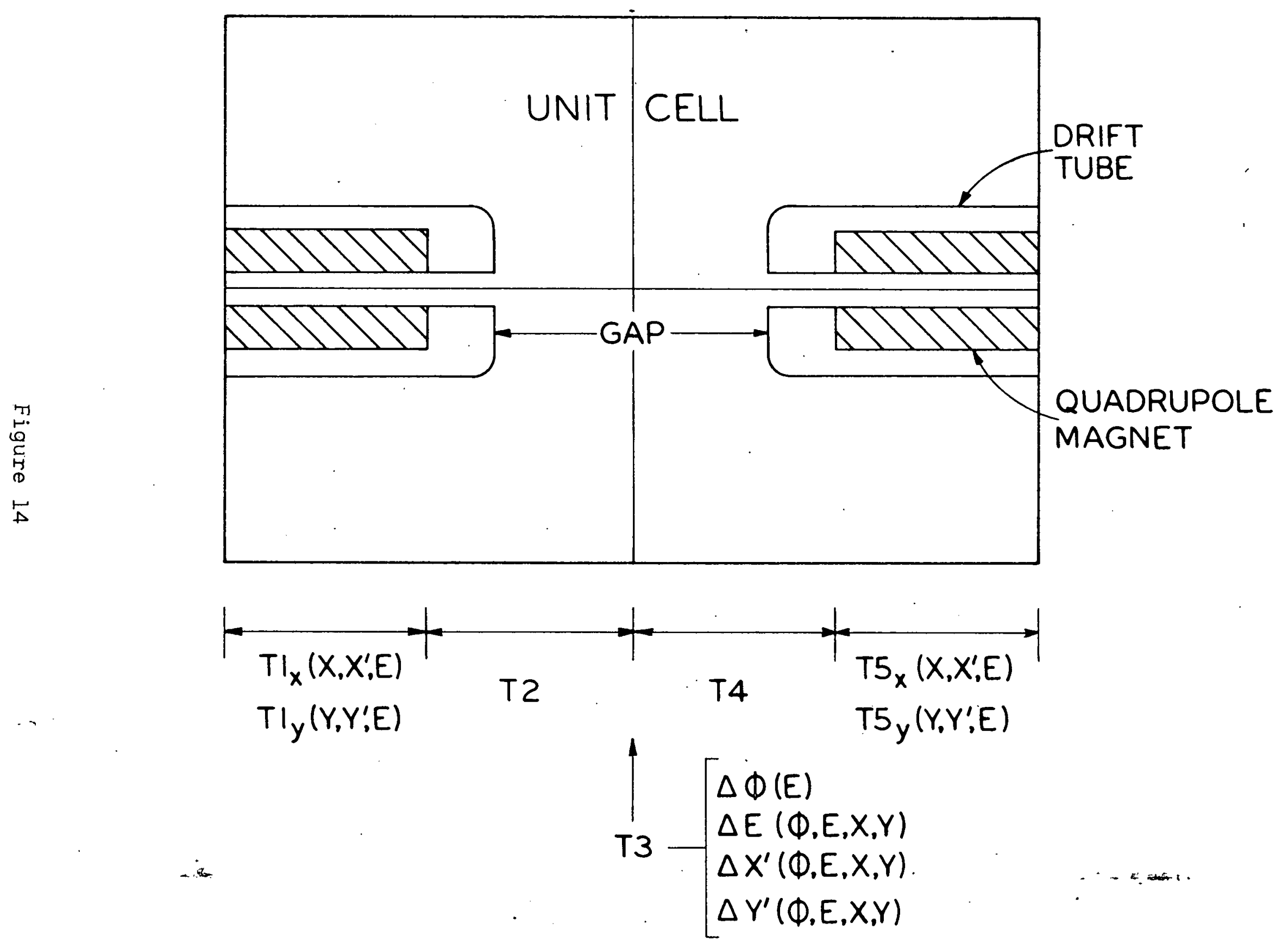

FUNCTIONAL DEPENDENCE OF TRANSFORMATION 